\title{
Calculation of the Reactance for a Magnetic Phase Created in a Steam Generator Tube Material
}

\author{
Kwon-sang Ryu ${ }^{1}$, Jae-Kap Jung ${ }^{2}$, Derac Son ${ }^{3}$, and Duck-gun Park ${ }^{4}$ \\ ${ }^{1}$ Div. of Industry Metrology, Korea Research Institute of Standards and Science, Daejeon 305-600, Korea \\ ${ }^{2}$ Div. of Physical Metrology, Korea Research Institute of Standards and Science, Daejeon 305-600, Korea \\ ${ }^{3}$ Department of Physics, Hannam University, Daejeon 300-791, Korea \\ ${ }^{4}$ Nuclear Materials Technology Development Team, KAERI, Daejeon 305-600, Korea
}

(Received 18 February 2010, Received in final form 6 April 2010, Accepted 9 April 2010)

\begin{abstract}
A magnetic phase is partly produced in a steam generator tube due to stress and heat, because steam generator tubes are exposed to high temperature, high pressure and radioactivity conditions. This adversely affects the safety of steam generator tubes. However, it is difficult to detect it using conventional eddy current methods. Therefore, a new type of probe is needed to separate the signals from the defects and magnetic phases. In this study, a new $U$-type yoke, which contained two types of coils, a magnetizing coil and detecting coil, was designed. In addition, the signal induced by the magnetic phase and defect in an Inconel 600 plate were simulated.
\end{abstract}

Keywords : FEM, magnetic phase (MP), steam generator tube (SGT), inconel 600

\section{Introduction}

Steam generator tubes (SGTs) in nuclear power plants transfer the heat created by fission in a primary loop to the turbine in a secondary loop. SGTs are produced from Inconel 600 series, which are non-magnetic. However, a magnetic phase (MP) is partly produced in the steam generator by stress and heat because the SGTs are exposed to high temperature, high pressure and radioactivity conditions [1,2]. This causes problems regarding the safety of SGTs but it is difficult to detect the MP using conventional eddy current techniques (ECTs).

Multi-frequency EC inspection techniques are among the most widely used techniques for rapid inspections of SGTs in nuclear power plants [3]. The ECT is applied to nonferrous materials with a relative permeability of 1 , such as Inconel alloy, because the magnetic permeability of magnetic materials severely limits the penetration depth of the induced ECs. Although the ECT is a widely used technique in the nuclear industry, it has a limitation in determining the size of a metal-loss defect accurately because the EC signal behavior depends on the total

*Corresponding author: Tel: +82-42-868-5164

Fax: +82-42-868-5018, e-mail: ryuks@kriss.re.kr defect volume. Furthermore, permeability variation clusters, such as the build-up of magnetite $\left(\mathrm{Fe}_{3} \mathrm{O}_{4}\right)$ and a magnetic phase on the secondary side of the SGT has no direct effect on the tube integrity but it causes spurious EC test results [4]. The relative permeability of a MP is $>1$, and a value of several thousand can be reached using a number of ferromagnetic metals.

Internal stresses caused by drawing, straightening and similar working of the material create a MP in the SGT, which can result in severe fluctuations in permeability. These fluctuations can always interfere with the EC test signals. Since the ferromagnetic test piece of MP is saturated by a strong magnetic field, a suitable device, such as magnetized ECT probe is employed to eliminate this interference effect during testing. The magnetic properties of the ferromagnetic test piece are similar to those of a non ferromagnetic material. Hence, the interference from permeability fluctuations is eliminated. Recently, a magnetized probe with a built-in permanent magnet was used in the SGT inspection to eliminate EC signal fluctuations because the strong magnetic field of this probe reduces the variations in magnetic permeability, which achieves a high $\mathrm{S} / \mathrm{N}$ ratio. However, the magnetized ECT probe could not saturate strong MP with large permeability, and the strong magnetic field of magnetized ECT probe amplifies the 
ECT signal with noise through the interaction of a magnetic field of the ECT probe and induces currents in the probe coil. The reliability of EC in a SGT inspection will be greatly enhanced if the MP can be separated from the defects selectively using a magnetic sensor.

In this study, a new probe was designed with a $U$-type yoke and two types of coils, a magnetizing coil and a detecting coil. FEM was used to simulate the reactance by FEM induced by the MP and a defect in an Inconel 600 plate.

\section{The Principle of the Designed Sensor}

The measuring principle of a MP in Inconel alloy is based on the change in magnetic flux density of a sample, the probe for measuring it composed of a $U$-type yoke wound magnetizing coil and $B$-sensing coil, and $H$-sensing coil, as shown in Fig. 1(a). The appearance of a MP in an Inconel alloy transforms the flow of magnetic flux through it because the EC is changed by the permeability of the MP. A change in flux density due to a MP can be obtained by measuring the eddy current in the yoke by the $B$-sensing coil. The probe shown in Fig. 1(b) consists of an exciting coil, $B$-sensing and $H$-sensing coil. This probe detects the voltage variations induced by the impedance change caused by the presence of a MP and a metal-loss defect in the structure under test.

\section{The Model}

Fig. 2(a), (b) and (c) shows the dimension of the designed probe, FEM model and mesh configuration of the MP and the defect, respectively. The length, width and thickness of the Inconel plate for the simulation were $100 \mathrm{~mm}, 20 \mathrm{~mm}$, and $3 \mathrm{~mm}$, respectively. The EC was solved using Ansoft Maxwell 11.1 3D software [5], because the $U$-type probe has two kinds of coils standing perpendicularly on the Inconel plate, including a MP and defect. The solver of the FEM was the EC, the number of meshes was 118,000 tetrahedra, and the solving frequency was 10 $\mathrm{kHz}$. The total current flowing in the magnetizing coil was $10 \mathrm{~A}$.

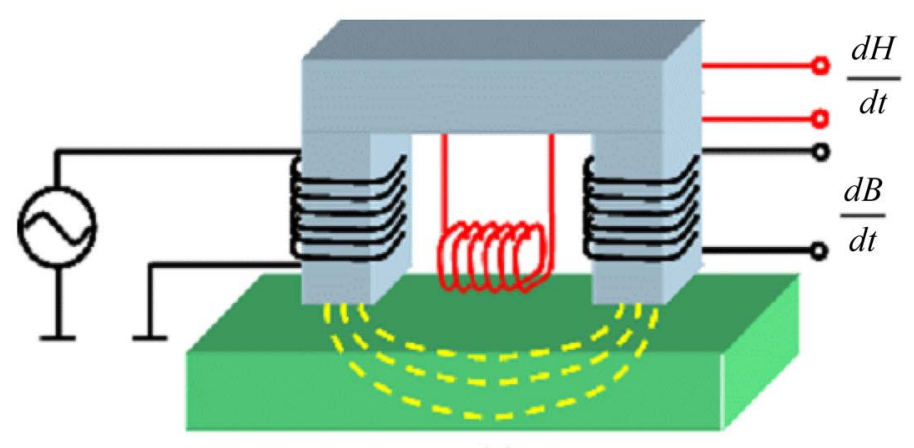

(a)

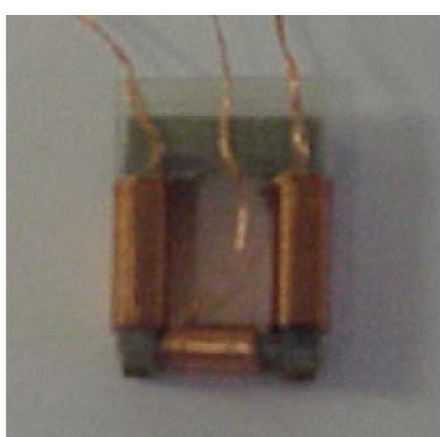

(b)

Fig. 1. (a) Schematic diagram of the $U$-type sensor for detecting the MP and defect, and (b) photograph of the developed sensor.
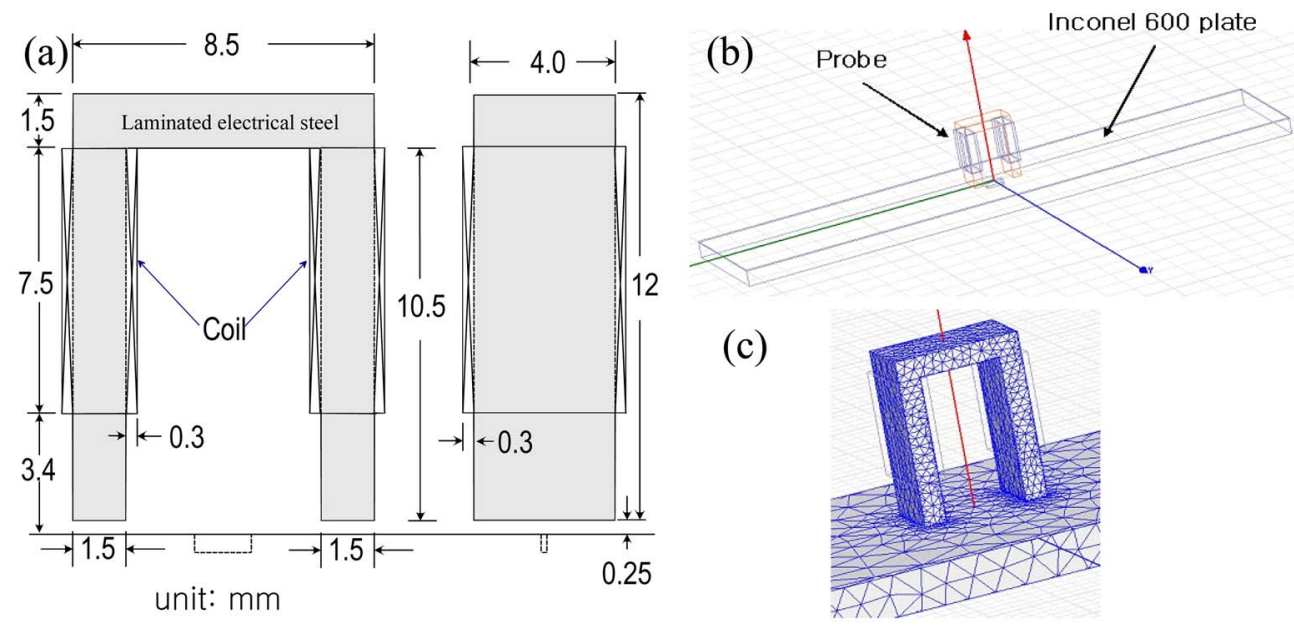

Fig. 2. (a) Dimension of probe, (b) FEM model, and (c) meshes configuration for simulating the EC. 


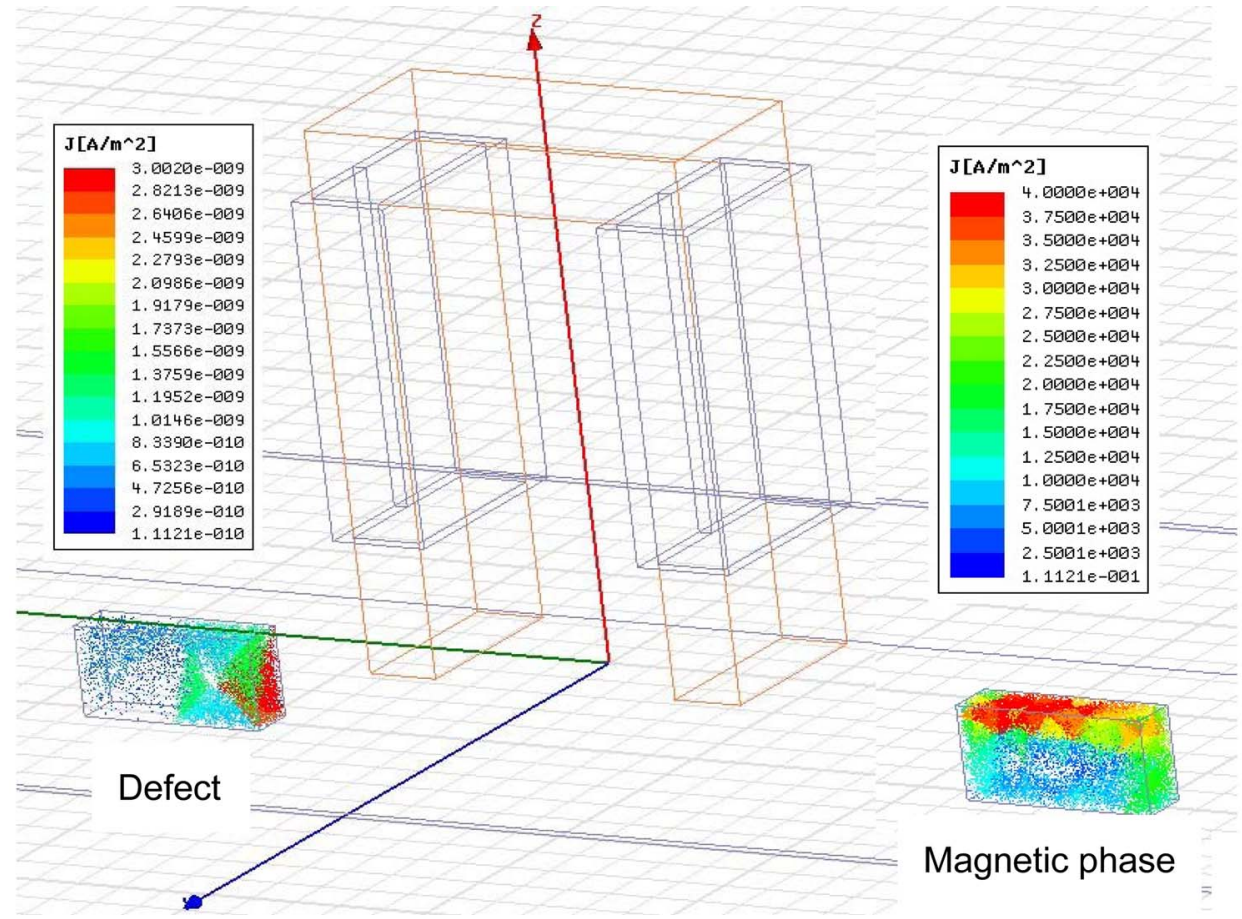

Fig. 3. EC configuration at the MP and defect.

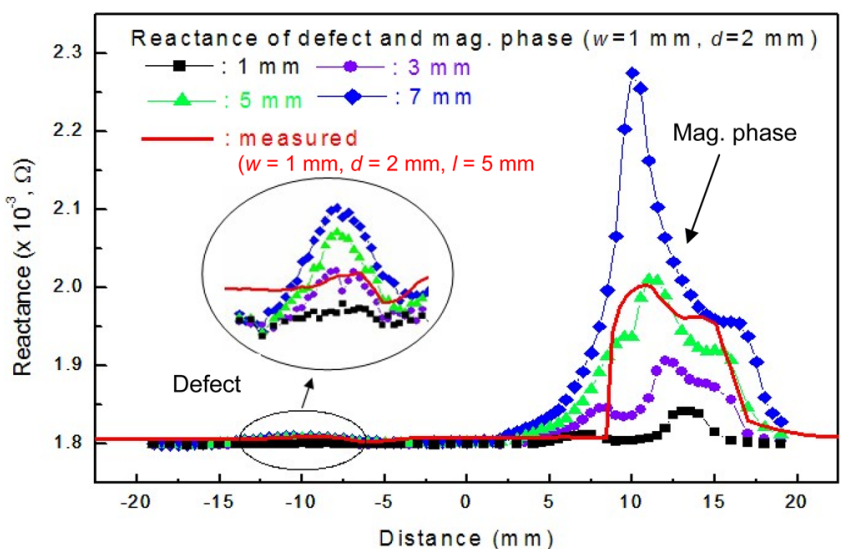

Fig. 4. (color online) Reactance calculated at the different lengths of the MPs and defect. The solid line without the symbols shows the measured result.

\section{Results and Discussions}

Fig. 3 illustrates the EC configuration on the MP and defect. The EC was stronger on the MP than the defect. The EC is composed of resistance (real part) and reactance (imaginary part). From the calculated result, the reactance is approximately 10 times higher than the resistance. Hence, the reactance was calculated. Fig. 4 shows the reactance changes according to the length of the MP and defect. The reactance amplitude increased with increas- ing length of the defect and MP, and was much stronger on the MP than the defect. The solid line without symbols indicates the reactance measured [6]; the trend of the simulated signal was similar to the measured one.

Fig. 5 shows the change in reactance for the depth (a) and width (b) of the MP and defects. The reactance increased with increasing depth and width of the MP and defect. Fig. 6 presents the change in reactance of the MP including defect in the center of a MP cluster. The calculated reactance at the MP with the defect decreased with increasing defect length. The magnetic permeability decreased with increasing defect length, so the eddy current also decreased because the EC is proportional to the magnetic permeability.

\section{Conclusions}

The reactance was simulated by FEM to design a new probe for detecting MPs and defects. The calculated reactance on the MPs and defects in an Inconel 600 plate agreed with the experimental results from the developed probe. The reactance increased with increasing depth and width of the MP and defect. The calculated reactance of the MP with the defect in the Inconel 600 plate decreased with increasing defect length. Overall, the FEM can be used to optimize the probe detecting the MP in an Inconel tube. 


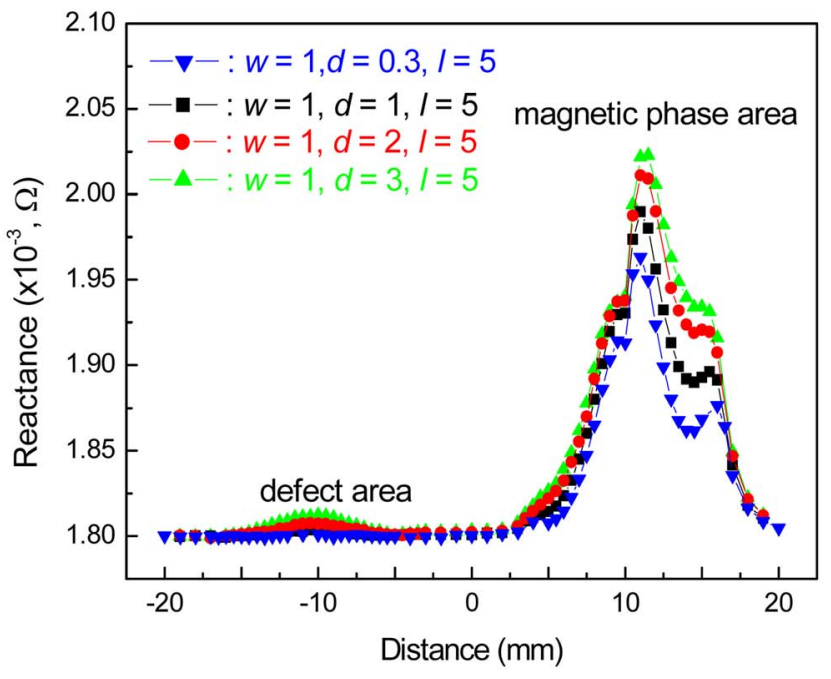

(a)

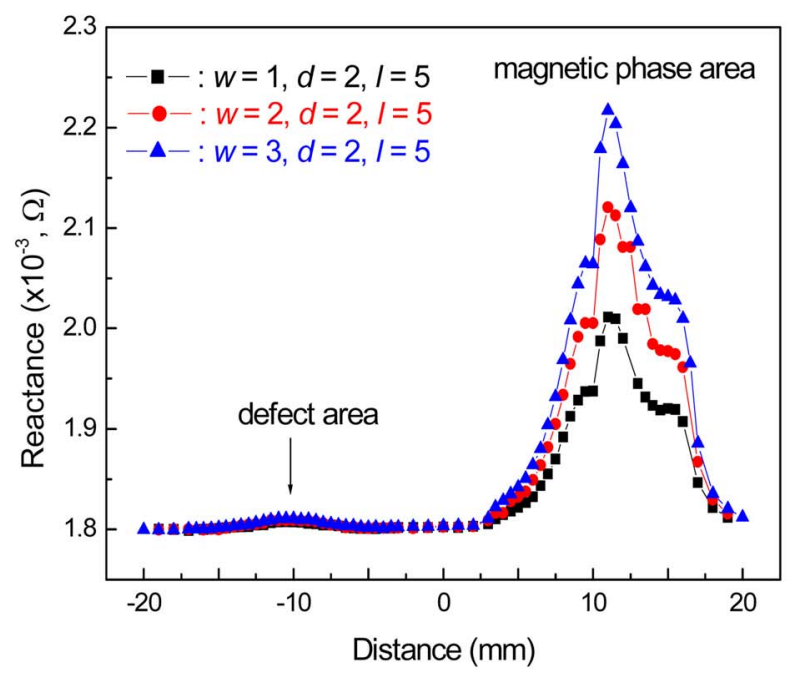

(b)

Fig. 5. (a) Reactance variation for (a) depth and (b) width of the MP and defects. $w$ is the width, $d$ is the depth, and $l$ is the length of the defect and MP.

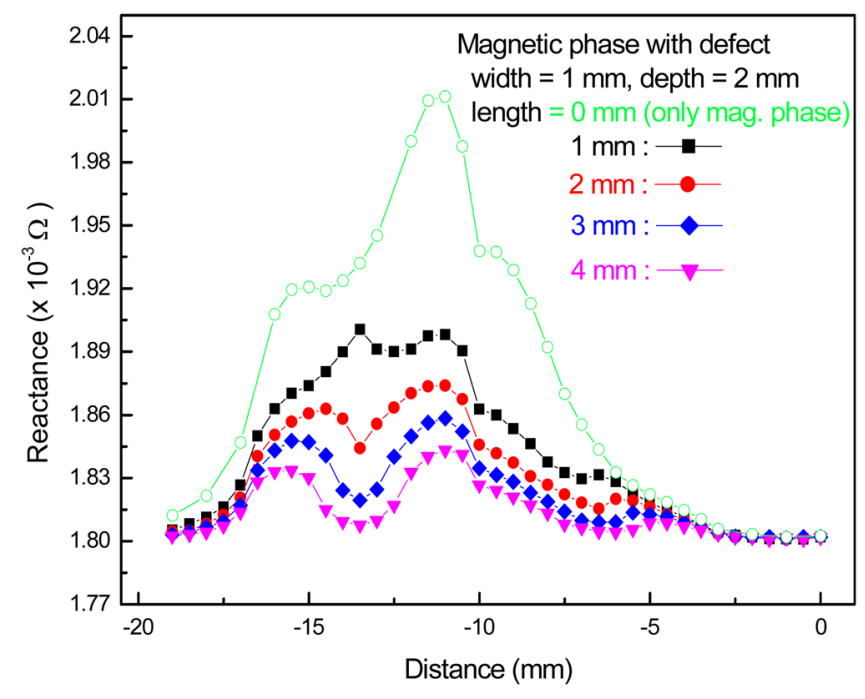

Fig. 6. Change in reactance at the MP including the different defect lengths in the center of a MP cluster.

\section{References}

[1] P. Xiang, et al., Int. J. Appl. Electromagnetics and Mechanics 12, 151 (2000).

[2] Y. Kamada, S. Takahashi, I. G. Park, et al., The $2^{\text {nd }}$ Asian Forum on Magnetics \& KMS 2005 Winter Conference, 6 (2005).

[3] V. S. Cecco, Eddy Current Manual, Chalk River National Laboratories, Ontario (1983).

[4] Young H. Kim, et al., Key Engineering Materials 270-273, 555 (2004).

[5] Ansoft Corp., http://www.ansoft.com

[6] D. G. Park, K. S. Ryu, and D. Son, Trans. Kor. Nuclear Soc. Spring Meeting, Chuncheon, Korea, May 25-26 (2006). 